

CARDIOLOGÍA DEL ADULTO - PRESENTACIÓN DE CASOS

\title{
Oclusión iatrogénica de la arteria coronaria derecha durante sustitución valvular tricuspídea
}

\section{Consuelo Alejandra Gotor Pérez*, Jorge Enrique Centeno Rodríguez, Enrique Pérez de la Sota, María Jesús López Gude y José María Cortina Romero}

\author{
Servicio de Cirugía Cardiaca, Hospital Universitario 12 de Octubre, Madrid, España
}

Recibido el 14 de febrero de 2018; aceptado el 15 de abril de 2018

Disponible en Internet el 1 de junio de 2018

\section{PALABRAS CLAVE \\ Sustitución valvular; Prótesis valvular; Complicaciones}

\section{KEYWORDS}

Valve replacement;

Valvular prosthesis;

Complications

\begin{abstract}
Resumen Se describe el caso de una mujer de 68 años que presentaba insuficiencia tricuspídea severa con ventrículo derecho dilatado, función sistólica levemente deprimida y ventrículo izquierdo no dilatado con fracción de eyección del 47\%. Se intervino mediante cirugía realizándose una sustitución valvular tricúspide por prótesis mecánica ATS n 33 e implante de electrodo de marcapasos epicárdico definitivo. En el postoperatorio inmediato presentó ascenso persistente del segmento ST en la cara inferior. Se implantó balón de contrapulsación intraaórtico y en el ecocardiograma urgente se observó disfunción ventricular global con aquinesia de la cara inferior. Se realizó coronariografía urgente observándose una imagen de angulación y deformidad a nivel distal de la arteria coronaria derecha no presente en la coronariografía prequirúrgica que sugería tracción externa del vaso, probablemente en relación con la sutura quirúrgica. Se intervino en forma percutánea implantándose stent farmacoactivo con lo cual se recuperó el flujo distal y se normalizó el segmento ST. La proximidad del anillo tricúspide a estructuras anatómicas como la arteria coronaria derecha hace posible su lesión durante la cirugía. El daño iatrogénico de la arteria coronaria derecha requiere diagnóstico y tratamiento precoz. Por ello esta complicación se debe incluir en el diagnóstico diferencial de disfunción ventricular derecha tras cirugía cardiaca.

(C) 2018 Sociedad Colombiana de Cardiología y Cirugía Cardiovascular. Publicado por Elsevier España, S.L.U. Este es un artículo Open Access bajo la licencia CC BY-NC-ND (http:// creativecommons.org/licenses/by-nc-nd/4.0/).
\end{abstract}

latrogenic occlusion of the right coronary artery during tricuspid valve replacement

Abstract The case is presented on a 68 year-old woman with severe tricuspid insufficiency. She also had a dilated right ventricle, a slightly depressed systolic function, and an undilated left ventricle with an ejection fraction of $47 \%$. We treated her surgically, the tricuspid valve replacement was carried out with an ATS $N^{\circ} 33$ mechanical prosthesis and implanted a

\footnotetext{
* Autor para correspondencia.

Correo electrónico: consuelogotorp@gmail.com (C.A. Gotor Pérez).
} 
permanent epicardial pacemaker lead. In the immediate post operative period, she presented a persistent ST segment elevation on the inferior wall. An intra-aortic balloon pump was implanted; the urgent echocardiogram showed a global ventricular dysfunction with akinesia of the inferior wall. An urgent coronary angiography was performed, with an image of angulation and deformity being observed at distal level of the right coronary artery that was not present in the pre-surgical coronary angiography, which suggested an external traction of the vessela probably associated with a surgical suture. Percutaneous intervention was carried out, with a drug-eluting stent being implanted. It was percutaneously treated by implanting a drug-eluting stent restoring distal blood flow and normalizing the ST segment. The proximity of the tricuspid ring to anatomical structures like the right coronary artery means that it could be damaged during surgery. The iatrogenic damage to the right coronary artery requires an early diagnosis and treatment. For this reason, this complication must be included in the differential diagnosis of right ventricular dysfunction after cardiac surgery.

(c) 2018 Sociedad Colombiana de Cardiología y Cirugía Cardiovascular. Published by Elsevier España, S.L.U. This is an open access article under the CC BY-NC-ND license (http:// creativecommons.org/licenses/by-nc-nd/4.0/).

El interés en la cirugía tricuspídea ha aumentado al conocer mejor la fisiopatología de la enfermedad tricuspídea. La insuficiencia tricuspídea puede clasificarse en primaria (orgánica) o secundaria (funcional); esta última es la etiología más frecuente.

En la afectación primaria existe alteración de los velos y/o aparato subvalvular acompañada o no de dilatación del anillo tricuspídeo. La etiología orgánica más común es la reumática y puede manifestarse como estenosis o insuficiencia.

En la afectación secundaria los velos son normales desde el punto de vista morfológico, pero existe una dilatación del anillo tricuspídeo y del ventrículo derecho (VD) que altera la relación espacial entre velos, cuerdas y músculos papilares, que hace que no coapten de manera adecuada. Suele ser secundaria a afectación del lado izquierdo del corazón, en especial por insuficiencia o estenosis mitral.

La decisión de tratar esta patología se basa en las consecuencias hemodinámicas y funcionales de la misma ${ }^{1}$.

La proximidad del anillo tricuspídeo a estructuras anatómicas como la arteria coronaria derecha (CD) o el sistema de conducción atrio-ventricular (AV), hace posible su lesión durante la cirugía ${ }^{2}$. De hecho, los estudios anatómicos de Berdajs et al. identificaron dos variantes morfológicas de la arteria del nodo $\mathrm{AV}$ en relación con la válvula tricúspide (VT), que aumentan el riesgo de lesionar la arteria y por ende producir alteraciones en la conducción AV. En un $40 \%$ de los casos la arteria transcurría por el centro del espacio comprendido entre las válvulas mitral y tricúspide, mientras que en otro $18 \%$ la arteria transcurría en íntima relación con el anillo del velo septal de la $\mathrm{VT}^{3,4}$.

Existe la posibilidad de lesionar la CD durante la cirugía tricuspídea debido a variaciones anatómicas de la coronaria, alteraciones de la relación entre la CD y el anillo tricuspídeo por dilatación de este último o remodelado ventricular ${ }^{2}$.

El daño iatrogénico de la CD es una rara complicación que tiene graves consecuencias si no se diagnostica y trata precoz y adecuadamente ${ }^{5}$. La CD surge del seno de Valsalva derecho, atraviesa el surco AV derecho en la proximidad de la VT hasta alcanzar la crux cordis. Cerca del velo posterior de la VT pasa el segmento medio-distal de la CD. Esta cercanía es mayor cuando el anillo se encuentra dilatado ${ }^{5}$, siendo precisamente esta la porción que más se dilata respecto a su diámetro inicial (hasta un $80 \%)^{6}$.

La CD durante la cirugía puede verse comprometida directamente por la sutura o indirectamente al tensar con la misma los tejidos situados en su proximidad. Parece ser más frecuente en pacientes con dominancia derecha coronaria, localizándose normalmente la lesión a nivel medio-distal. Si la oclusión es completa los signos de isquemia miocárdica pueden presentarse intraoperatoriamente, mientras que la oclusión incompleta puede manifestarse horas o días después de la cirugía 5 .

Los cambios transitorios en el electrocardiograma (ECG) y las alteraciones del movimiento en la pared ventricular postcirculación extracorpórea, suelen atribuirse a embolia aérea, lo cual dificulta el diagnóstico ${ }^{5}$. Sin embargo, la inestabilidad hemodinámica o eléctrica con alteraciones de la contractilidad ventricular (disfunción) derecha o biventricular y los cambios agudos en el segmento ST, deben hacer sospechar lesión de la CD. El diagnóstico diferencial debe incluir otras causas de disfunción ventricular derecha como sobrecarga de volumen, hipertensión pulmonar o embolia aérea ${ }^{2}$.

Cuando el diagnóstico se realiza intraoperatoriamente se puede retirar el material de sutura (no asegura la resolución si la lesión de la CD es directa) o realizar un bypass distal en dicha arteria. Si el diagnóstico se efectúa en el postoperatorio precoz se debe realizar una coronariografía emergente para localizar el daño y reestablecer el flujo coronario. Si la intervención percutánea falla se debe intervenir quirúrgicamente ${ }^{5}$.

Se presenta el caso de una mujer de 68 años con antecedente personal de sustitución valvular mitral por prótesis mecánica en el año 2000 y colocación de stent convencional en la CD proximal en el año 2007. Como factores de riesgo cardiovascular presentaba diabetes mellitus, hipertensión 




Figura 1 La flecha señala la zona de lesión en la coronaria derecha.

arterial y dislipidemia. Se encontraba en fibrilación auricular (FA) lenta a 40 latidos por minuto. Estaba en seguimiento por insuficiencia tricuspídea severa. En el ecocardiograma la VT presentaba velos restrictivos, de aspecto reumático, además se observaba VD dilatado con función sistólica levemente deprimida y ventrículo izquierdo (VI) no dilatado con fracción de eyección (FE) del $47 \%$ e hipoquinesia moderada del segmento medio-basal de la cara septal. Se encontraba en clase funcional (CF) III/IV de la New York Heart Association (NYHA). La coronariografía preoperatoria mostraba dominancia derecha sin lesiones coronarias significativas y sin reestenosis del stent. Se decide intervenir quirúrgicamente realizándose una sustitución valvular tricúspide (SVT) por prótesis mecánica ATS n॰33 e implante de electrodo de marcapasos epicárdico definitivo de manera profiláctica, en cara diafragmática de VD, dados su antecedente de FA lenta y mayor incidencia de bloqueo AV con la SVT ${ }^{4,7}$. En el postoperatorio inmediato presentó ascenso persistente del segmento ST en cara inferior. Se decidió implante de balón de contrapulsación intraaórtico y ecocardiograma urgente donde se observó disfunción ventricular global con aquinesia de cara inferior. Tras aproximadamente una hora en la Unidad de Cuidados Intensivos, se hizo coronariografía urgente que mostró imagen de angulación y deformidad a nivel distal de la CD no presente en la coronariografía prequirúrgica, que sugería tracción externa del vaso, probablemente en relación con la sutura quirúrgica (fig. 1). Se cruzó dicha zona con guía Pilot 50 y con guía BMW, se predilató con balón de 1,2 y $2 \mathrm{~mm}$ y se implantó stent farmacoactivo de $3 \times 20 \mathrm{~mm}$ recuperando flujo distal TIMI (thrombolysis in myocardial infarction) 3 tanto en la rama descendente posterior como en la rama posterolateral (fig. 2). Tras la intervención percutánea se normalizó el segmento ST. Respecto a los marcadores de daño miocárdico los valores máximos de CPK (creatina fosfoquinasa), CPK-MB (creatina fosfoquinasa fracción $\mathrm{MB}$ ) y troponina $\mathrm{T}$ hs se alcanzaron a las 12 horas del ingreso en la Unidad de Cuidados Intensivos y fueron 1.781

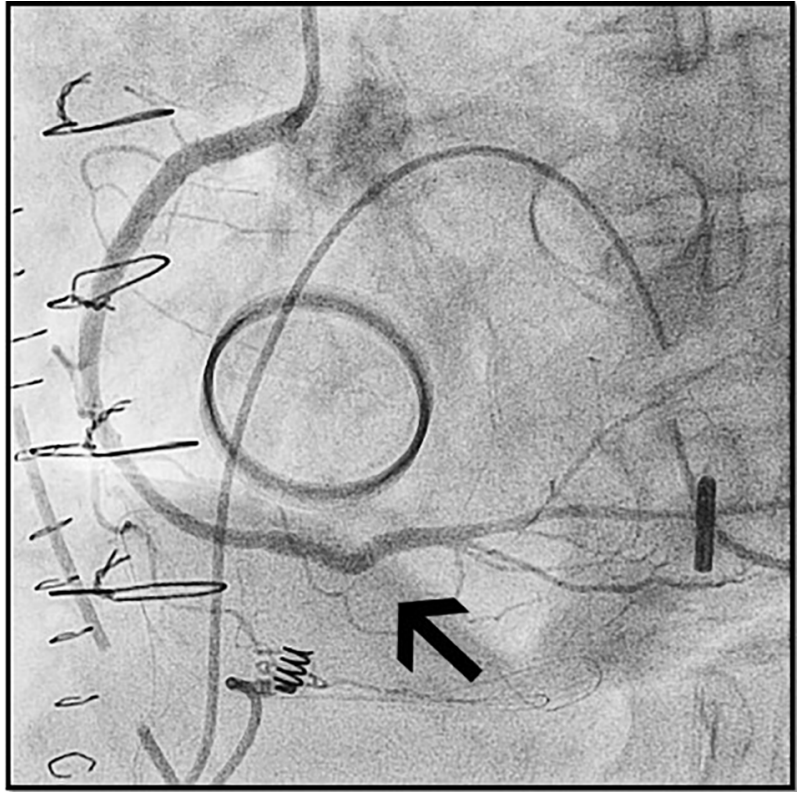

Figura 2 Se observa cómo se ha restaurado el flujo en la arteria coronaria.

$\mathrm{U} / \mathrm{l}, 175,4 \mathrm{ng} / \mathrm{ml}$ y $13.415 \mathrm{ng} / \mathrm{l}$ respectivamente. El ecocardiograma postoperatorio objetivó un VI no dilatado con FE del $40 \%$ e hipoquinesia del septo, inferior y anterior; VD no dilatado con disfunción sistólica leve-moderada y prótesis tricúspide normofuncionante. El resto de la estancia hospitalaria transcurrió sin incidencias y la paciente fue dada de alta a los 17 días postoperatorios sin secuelas y en proceso de rehabilitación. En la revisión postoperatoria a los tres meses de la intervención quirúrgica, se encontraba en clase funcional II/IV de la NYHA y en el ecocardiograma de control se observó VI no dilatado con FE del 39\% con hipoquinesia marcada en cara inferior e inferolateral, VD dilatado con función sistólica severamente deprimida, prótesis mecánicas en posición tricúspidea y mitral normofuncionantes y signos indirectos de hipertensión pulmonar.

La lesión de la CD durante la cirugía tricuspídea es una complicación rara, posiblemente infradiagnosticada, que requiere diagnóstico y tratamiento precoz para evitar las graves consecuencias que puede conllevar. Por tanto, es crucial incluir esta lesión en el diagnóstico diferencial de disfunción ventricular derecha tras excluir otras causas más frecuentes.

\section{Conflicto de intereses}

Ninguno.

\section{Bibliografía}

1. Shinn SH, Schaff HVI. Evidence-based surgical management of acquired tricuspid valve disease. Nat Rev Cardiol. 2013;10:190-203.

2. Díez-Villanueva P, Gutiérrez-Ibáñez E, Cuerpo-Caballero GP, Sanz-Ruiz R, Abeytua M, Soriano J, et al. Direct injury to right coronary artery in patients undergoing tricuspid annuloplasty. Ann Thorac Surg. 2014;97:1300-5. 
3. Berdajs D, Schurr UP, Wagner A, Seifert B, Turina MI, Genoni M. Incidence and pathophysiology of atrioventricular block following mitral valve replacement and ring annuloplasty. Eur J Cardiothorac Surg. 2008;34:55-61.

4. Mestres CA, Suri RM. Pacemaker risk associated with prophylactic tricuspid annuloplasty: Balancing beneficence and nonmaleficence. J Thorac Cardiovasc Surg. 2016;151:104-5.

5. Smith $\mathrm{BB}$, Smith $\mathrm{MM}$, Rehfeldt $\mathrm{KH}$. latrogenic right coronary artery occlusion following tricuspid valve repair: case report of a rare but recognized complication. A\&A Case Reports. 2017; 8:39-41.

6. Filsoufi F, Salzberg SP, Abascal V, Adams DH. Surgical management of functional tricuspid regurgitation with a new remodeling annuloplasty ring. Mt Sinai J Med. 2006;73:874-9.

7. Lewis JW Jr, Webb CR, Pickard SD, Lehman J, Jacobsen G. The increased need for permanent pacemaker after reoperative cardiac surgery. J Thorac Cardiovasc Surg. 1998;116:74-81. 